\title{
Pomegranate juice enhances healthy lifespan in Drosophila melanogaster: an exploratory study
}

\section{Subramani Paranthaman Balasubramani ${ }^{1,2}$, Jayaram Mohan ${ }^{3}$, Arunita Chatterjee $^{3}$, Esha Patnaik ${ }^{3}$, Subrahmanya Kumar Kukkupuni ${ }^{1,2}$, Upendra Nongthomba ${ }^{3}$ and Padmavathy Venkatasubramanian ${ }^{1}$ *}

\author{
${ }^{1}$ Foundation for Revitalisation of Local Health Traditions (FRLHT), Bangalore, India \\ 2 Manipal University, Manipal, India \\ ${ }^{3}$ Department of Molecular Reproduction, Development and Genetics, Indian Institute of Science, Bangalore, India
}

\section{Edited by:}

Anwar Huq, University of Maryland,

USA

Reviewed by:

Juan M. Gonzalez, Consejo Superior de Investigaciones Científicas, Spain Gulnihal Ozbay, Delaware State

University, USA

Salina Parveen, University of

Maryland Eastern Shore, USA

*Correspondence:

Upendra Nongthomba, Department

of Molecular Reproduction,

Development and Genetics, Indian

Institute of Science, Bangalore

560012, India

e-mail: upendra@mrdg.iisc.ernet.in;

Padmavathy Venkatasubramanian,

Foundation for Revitalisation of Local

Health Traditions (FRLHT), 74/2,

Jarakabande Kaval, Attur Post, Via

Yelahanka, Bangalore 560106, India

e-mail: padma.venkat@frlht.org
Exploring innovative ways to ensure healthy aging of populations is a pre-requisite to contain rising healthcare costs. Scientific research into the principles and practices of traditional medicines can provide new insights and simple solutions to lead a healthy life. Rasayana is a dedicated branch of Ayurveda (an Indian medicine) that deals with methods to increase vitality and delay aging through the use of diet, herbal supplements, and other lifestyle practices. The life-span and health-span enhancing actions of the fruits of pomegranate (Punica granatum L.), a well-known Rasayana, were tested on Drosophila melanogaster (fruitfly) model. Supplementation of standard corn meal with $10 \%(\mathrm{v} / \mathrm{v})$ pomegranate juice (PJ) extended the life-span of male and female flies by 18 and $8 \%$, respectively. When male and female flies were mixed and reared together, there was $19 \%$ increase in the longevity of PJ fed flies, as assessed by MSD, the median survival day (24.8). MSD for control and resveratrol (RV) groups was at 20.8 and 23.1 days, respectively. A two-fold enhancement in fecundity, improved resistance to oxidative stress $\left(\mathrm{H}_{2} \mathrm{O}_{2}\right.$ and paraquat induced) and to Candida albicans infection were observed in PJ fed flies. Further, the flies in the PJ fed group were physically active over an extended period of time, as assessed by the climbing assay. PJ thus outperformed both control and RV groups in the life-span and health-span parameters tested. This study provides the scope to explore the potential of $P J$ as a nutraceutical to improve health span and lifespan in human beings.

Keywords: pomegranate, anti-aging, rasayana, ayurveda, Drosophila

\section{INTRODUCTION}

Global statistics predict $223 \%$ increase in the number of elderly (aged $>60$ years) accounting for about 1.2 billion between 1970 and 2025 (1). Ways to ensure healthy aging while increasing lifespan are heatedly debated and researched in public health circles (2). This is because long-term institutional care of the elderly for chronic age-related conditions such as arthritis and dementia has led to steep increases in healthcare costs, yet reduced the quality of life for the patients $(3,4)$. Countries have started recognizing that promoting self-reliant wellness strategies among communities would not only save the exchequer from substantial financial burden due to illness care but also have a population that is healthy and productive (5). Increasing evidence through longitudinal studies show that simply adopting appropriate diet and lifestyle can even reverse chronic conditions like coronary atherosclerosis (6).

Complementary and alternative medicines like ayurveda, yoga, and traditional Chinese medicine prescribe several interventions for prevention of diseases and promotion of health that can be scientifically explored for wellness. Rasayana is a special branch of Ayurveda, which deals with methods of rejuvenation such as dietary recipes and regimen, herbal and mineral supplements, and health-promoting lifestyle that are said to enhance quality of life and delay aging (7). Traditional literature describes Rasayanas as methods of reversing naturally occurring senility and improving mental competence (8), increasing immunity against diseases and providing vitality and luster to the body (7). As per Ayurveda, Rasayanas act on the body by (i) improving the process of digestion and metabolism (Agni), leading to better bio-availability, (ii) increasing the micro-circulation of nutrients through the body channels (Srotas), and (iii) enhancing the nutritive value of the plasma that gets generated (Rasadhatu) $(7,9)$. Several studies have indicated Rasayanas to be anti-oxidants and immunomodulators (10).

Ayurveda identifies several fruits and herbs including the Indian Gooseberry (Phyllanthus emblica), Pomegranate (Punica granatum), Drumsticks (Moringa oleifera), Long pepper (Piper longum), etc., as Rasayanas $(7,8,10)$. Understanding the scientific basis behind the concept of Rasayana and validation of the life promoting ability of commonly available Rasayana fruits and herbs can help establish holistic and simple ways to wellness through diet.

The potential therapeutic properties of pomegranate, a commonly available fruit, have been studied for wide-ranging applications including cardiovascular disease, diabetes, dental conditions, erectile dysfunction, protection from ultraviolet (UV) radiation, infant brain ischemia, prevention of cancer, Alzheimer's disease, 
male infertility, arthritis, and obesity (11-13). Pomegranate is considered as a symbol of life, health, longevity, fecundity, intellect, immortality, and spirituality by Unani and Traditional Chinese Medical systems as well (14). Ayurvedic texts describe pomegranate as a "wholesome" (Pathya) fruit with several properties including tissue generation and development and strength promotion (15). It is particularly indicated for use in cardiac and anemic conditions (16).

Aging is a complex physiological phenomenon, particularly because it manifests itself over a wide range of biological systems, tissues, and functions. Aging pathways are highly conserved across species including Saccharomyces cerevisiae, Caenorhabditis elegans, D. melanogaster, and rodents $(17,18)$. Drosophila (commonly known as fruit fly) has many advantages for studying the biology of aging. For example, flies show physiological, genetic, and anatomical similarities with human (17). Owing to short-lifespan and ease of handling, the fruit flies are particularly ideal for conducting prevention and wellness studies, which would otherwise take several years in human beings (17). Fruits like cranberry (19), blueberry (20), orange, lemon (21), apple polyphenols (22), and cocoa (23) have been shown to enhance lifespan in Drosophila model. A few Ayurvedic formulations have also been tested for their bioactive potential in the Drosophila model $(24,25)$.

Pomegranate, a member of the Punicaceae family is native to the Himalayas. However, it is also under commercial cultivation in other parts of India and the world (12). It contains polyphenols such as ellagitannins and anthocyanins, as well as phenolic acids, fatty acids, alkaloids, and a variety of volatile compounds (26). Ellagitannins are among the most prevalent compounds present in pomegranate and may be responsible for various bioactivities (27). The juice contains ellagic acid, gallic acid, anthocynidins, flavan3-ols, straight chain fatty acids, citric acid, and malic acid besides glucose, fructose, and sucrose as the major sugars (26). Pomegranate has been shown to have anti-oxidant, anti-inflammatory, lipid regulation, gastroprotective, anti-diarrhea, anti-cancer, anti-viral, anti-angiogenesis, anti-diabetic, and anti-atherosclerotic activities $(26,27)$.

The Rasayana actions of pomegranate, namely, enhancement of life-span, reproductive ability, stress tolerance, resistance to infection, and stamina were compared between control, resveratrol (RV), and pomegranate juice (PJ) supplemented D. melanogaster. Our hypothesis was that pomegranate supplementation would significantly improve the performance of the flies on all counts, as compared to the control.

\section{MATERIALS AND METHODS \\ DROSOPHILA STRAIN, MEDIA, AND CULTURE CONDITIONS}

Drosophila melanogastor wild type, Canton-S strain, was used for this study. The basal media for culturing the flies consisted of corn flour (60 g), sugar (20 g), D-glucose (20 g), dry yeast (15 g), and agar $(8 \mathrm{~g})$ per liter. After cooking, media was allowed to cool to room temperature and then propionic acid $(4 \mathrm{ml})$, benzoic acid $(0.7 \mathrm{~g}$ in $5 \mathrm{ml}$ of ethanol), orthophosphoric acid $(0.6 \mathrm{ml})$ were added and mixed thoroughly. Media cooked as slurry was poured into the bottles or vials and was allowed to solidify before transfer of adult flies or pupae. Stock flies were maintained in bottles while culture vials were used for maintenance of smaller populations or experimental groups. Flies were allowed to feed ad libitum and grown at $25^{\circ} \mathrm{C}$ with $60 \%$ humidity in $12 \mathrm{~h}$ day-night cycle.

\section{PREPARATION OF POMEGRANATE JUICE AND RESVERATROL STOCK}

Pomegranate juice was prepared by hand crushing the arils of fresh fruits through sterile muslin cloth. The juice was passed through a filter $(0.2 \mu \mathrm{m}$; Millex, Millipore, Germany) and stored as aliquots of different volume $(5,15$, and $50 \mathrm{ml})$ in screw capped tubes at $-80^{\circ} \mathrm{C}$ until use. Freezing did not significantly alter the highperformance liquid chromatography (HPLC) profile (data not shown). RV (Sigma-Aldrich, St. Louis, MO, USA) stock (100 mM) was prepared by dissolving $22.8 \mathrm{mg}$ in $1 \mathrm{ml}$ of ethanol. Both PJ and RV were added to the cooked and warm basal media before dispensing into vials.

\section{LIFESPAN EXTENSION EXPERIMENT}

Age matched (2-3 days) flies were generated by removing all adult flies in the bottles. Once a synchronous culture was generated, sex separated flies were transferred into vials containing basal medium supplemented with $0.1,1,5,10$, and $15 \%(\mathrm{v} / \mathrm{v})$ of PJ. Flies were transferred to fresh media every third day. Dead flies were counted and removed daily until the death of the last fly in each vial. PJ at $15 \% \mathrm{v} / \mathrm{v}$ could not be used since at that concentration the medium would not set.

\section{LONGEVITY AND FECUNDITY ANALYSIS}

Synchronous cultures of 2-3-day-old files were obtained as described earlier and transferred into vials containing basal medium supplemented with $10 \%$ of PJ (v/v) or $200 \mu \mathrm{M}$ RV and control. Each group including the control had 10 vials each with 14-20 flies per vial with equal sex ratio. Flies were transferred to fresh media every third day. Dead flies were counted and removed daily throughout the experiment. New flies emerging in the emptied vials were counted daily for 10 days from the day of the emergence of the first fly in each experimental group.

\section{GUSTATORY ASSAY}

The method described by Lee et al. (28) was adopted for gustatory assay. Adult flies were reared for 20 or 40 days in media supplemented with $10 \%$ PJ or $200 \mu \mathrm{M}$ RV. A basal media control was also maintained. To perform a feeding assay, after starving the flies for $2 \mathrm{~h}, 30$ male or female flies from each experimental group were transferred into the vials containing the specific diets with bromophenol blue dye $(0.05 \% \mathrm{wt} / \mathrm{vol})$ (Sigma-Aldrich, St. Louis, MO, USA). After $10 \mathrm{~min}$ of feeding, the fed flies were etherized, washed with phosphate-buffered saline (PBS), and homogenized in $1 \mathrm{ml}$ of distilled water. The absorbance of 100 times diluted supernatant was measured at $595 \mathrm{~nm}$ using a spectrophotometer (Bio-Rad, CA, USA).

\section{RESISTANCE AGAINST HYDROGEN PEROXIDE AND PARAQUAT INDUCED STRESS}

The method described by Peng et al. (22) was used for studying the fly's ability to resist free radical stress induced by $\mathrm{H}_{2} \mathrm{O}_{2}$ (Fisher Scientific, Mumbai, India) and paraquat (Sigma-Aldrich, St. Louis, MO, USA). $\mathrm{H}_{2} \mathrm{O}_{2}$ generates hydroxyl radical $\left(\mathrm{OH}^{\star}\right)$ while paraquat produces superoxide $\left(\mathrm{O}^{\star}\right)$ radicals. Twenty days 
old male and female flies from control, RV $(200 \mu \mathrm{M})$, and PJ (10\%) fed were starved for $2 \mathrm{~h}$ and transferred to separate vials $(n=50$; 10 flies/vial) containing $5 \% \mathrm{H}_{2} \mathrm{O}_{2}$ or $20 \mathrm{mM}$ paraquat prepared with $5 \%$ sucrose on saturated tissue paper mat. Dead flies were counted every $4 \mathrm{~h}$ until the death of the last fly.

\section{RESISTANCE AGAINST INFECTION}

The method described by Apidianakis and Rahme (29) was followed with slight modifications. Antigen was prepared by suspending an overnight culture of C. albicans SC5314 in sterile distilled water $\left(2 \times 10^{8}\right.$ cells $/ \mathrm{ml}$ - as counted using a hemocytometer; data not shown). Flies (20 days old) were anesthetized with $\mathrm{CO}_{2}$. Infection was induced with a sterile tungsten needle $(0.01 \mathrm{~mm}$ diameter $)$ dipped in the $C$. albicans suspension by gently pricking in the thoracic region. Flies were returned to their respective food vials and were incubated at $25^{\circ} \mathrm{C}$ with $12 \mathrm{~h}$ day-night cycle until $50 \%$ of flies died in any of the groups. Control flies were pricked with needle dipped in sterile distilled water.

\section{CLIMBING ASSAY}

On day 20 and 40, locomotor function of fruit flies was assessed using the climbing or negative geo-taxis assay as reported by Bahadorani and Halliker (23) with slight modifications. In brief, 20 flies/trial were placed in the bottom of a measuring cylinder and given $20 \mathrm{~s}$ to climb up. At the end of each trial, the number of flies that climbed up to a vertical distance of $>8 \mathrm{~cm}$ was recorded. Each trial was repeated three times.

\section{STATISTICAL ANALYSIS}

The day when $50 \%$ of the total flies in each of the experimental group survived was calculated as the median survival day. Log rank test ${ }^{1}$ was used for survival analysis while Wilcoxon rank sum test ${ }^{2}$ was used for comparing the life-span extension (30). Student's $t$-test was used for comparison of means. ANOVA was employed while comparing more than two groups. $p$-Value $<0.05$ was considered as significant.

${ }^{1}$ http://bioinf.wehi.edu.au/software/russell/logrank/

${ }^{2} \mathrm{http}: / /$ www.fon.hum.uva.nl/Service/Statistics/Wilcoxon_Test.html

\section{RESULTS}

\section{PJ SUPPLEMENTATION EXTENDS LIFESPAN IN DROSOPHILA}

To determine the lifespan extending potential in Drosophila, we supplemented the basal media with $0.1,1,5,10$, and $15 \%$ of PJ. Substitution of the media with $10 \%$ PJ significantly enhanced the median lifespan by 18.51 and $8 \%$ in male and female flies, respectively (Figure 1; Table 1). Last fly survival day was also enhanced by 2 days in the $10 \%$ PJ group. While the lower concentration tested $(0.1,1$, and $5 \%)$ did not produce any significant changes in the life-span, $15 \%$ PJ substitution created a practical difficulty in solidification of the medium; therefore, this concentration was not considered. Thus, $10 \% \mathrm{PJ}$ was used in all further experiments.

\section{PJ SUPPLEMENTATION CONTROLS THE "TRADE-OFF" BETWEEN FECUNDITY AND LONGEVITY}

As $10 \%$ PJ substitution to the media significantly enhanced lifespan for both male and female flies separately, we investigated the effect of this concentration in the mixed group of male and female. RV at $200 \mu \mathrm{M}$ dose was used as a positive control, based on the reports of Bass et al. (31).

Pomegranate juice enhanced the median survival of a mixed population of flies from 20.8 (as seen in control) to 24.8 days (PJ), i.e., PJ enhanced median life-span by $19.23 \%$ more than the control $(p<0.00001)$ (Figure 2A; Table 1). RV group on the other hand enhanced the median survival to 23.1 days, which was only $11 \%$ over the control group (Figure 2A; Table 1). The maximum life-span observed in the control and PJ groups was 61 days while that in the RV group, the last fly survived till 62nd day. Reproductive potential of an organism also indicates its physiological fitness. So, we monitored the number of flies emerging from each vial of PJ, RV, and control group. The data shown in Figure 2B indicate that PJ group produced significantly $(p<0.0001)$ more number of flies throughout the reproductive phase. Also, the new fly emergence data indicated that PJ extended the reproductive viability phase (Figure 2B).

\section{DROSOPHILA HAS THE SAME FEEDING PREFERENCE TO PJ AND RV SUPPLEMENTATION}

Feeding behavior and nutritional constituents of a culture medium are important factors in the life-span determination of
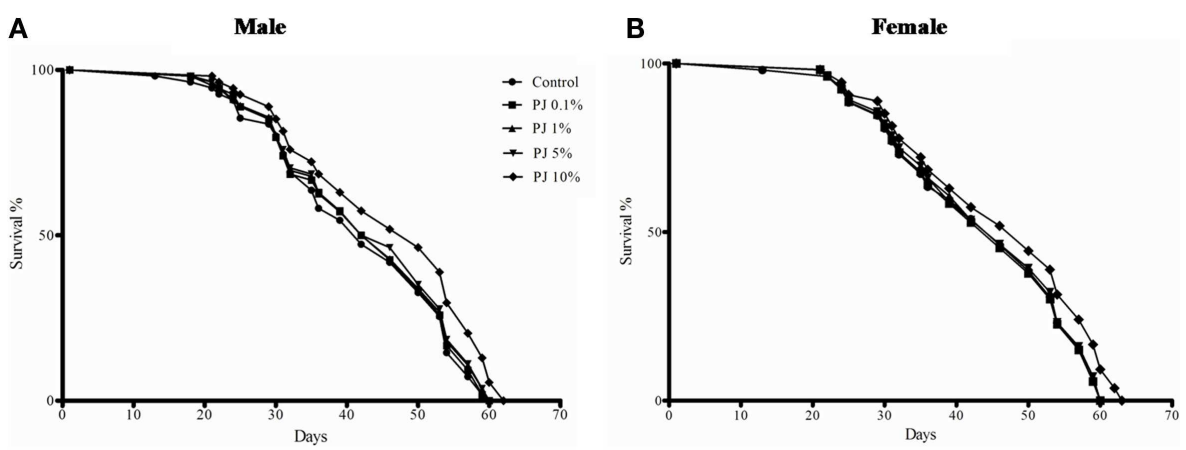

FIGURE 1 | Life-span extension by different concentrations of pomegranate juice (PJ) in Drosophila (A) male flies and (B) female flies. Ten percent PJ substitution to the media significantly increased $(p<0.00001)$ the survival of the flies and extended their life-span by 2 days. In both the sexes 0.1 , 1 , or $5 \%$ PJ did not show any enhancement in the survival. 
Table 1 | Statistical analysis of the survival curves using Log rank tests and Wilcoxon rank sum tests.

\begin{tabular}{|c|c|c|c|c|c|c|}
\hline $\begin{array}{l}\text { Experimental } \\
\text { group }\end{array}$ & $\begin{array}{c}\text { Number of flies } \\
\text { tested }(n)\end{array}$ & $\begin{array}{l}\text { Median survival } \\
\text { day/hour }\end{array}$ & $\%$ Change & $\begin{array}{c}\text { Chi square } \\
\text { value @ } 1 \text { degree } \\
\text { of freedom }\end{array}$ & $\begin{array}{l}\text { Log rank } \\
\text { (survival) }\end{array}$ & $\begin{array}{l}\text { Wilcoxon } \\
\text { (maximum } \\
\text { life-span) }\end{array}$ \\
\hline \multicolumn{7}{|l|}{ Male } \\
\hline Control & 110 & 40.5 & & & & \\
\hline PJ 0.1 & 108 & 42.0 & 3.7 & 0.7 & $p=0.396$ & $p \leq 0.6421$ \\
\hline PJ 5 & 108 & 42.0 & 3.7 & 3.9 & $p=0.047$ & $p \leq 0.6208$ \\
\hline PJ 10 & 108 & 48.0 & 18.51 & 46.2 & $p<0.00001^{*}$ & $p \leq 0.2545$ \\
\hline \multicolumn{7}{|l|}{ Female } \\
\hline Control & 104 & 43.5 & & & & \\
\hline PJ 0.1 & 106 & 43 & -1.14 & 0.1 & $p=0.699$ & $p \leq 0.6861$ \\
\hline Control & 247 & 20.8 & & & & \\
\hline $\mathrm{RV}$ & 167 & 23.1 & 11.05 & 16.2 & $p<0.00001^{*}$ & $p \leq 0.6016$ \\
\hline PJ & 164 & 24.8 & 19.23 & 48.7 & $p<0.00001^{*}$ & $p \leq 0.3506$ \\
\hline \multicolumn{7}{|c|}{$\mathrm{H}_{2} \mathrm{O}_{2}$ stress survival } \\
\hline \multicolumn{7}{|c|}{ Male } \\
\hline Control & 50 & 18.0 & & & & \\
\hline RV & 50 & 24.5 & 36.1 & 29.3 & $p<0.00001^{*}$ & $p \leq 0.4705$ \\
\hline PJ & 50 & 28.0 & 55.5 & 1.6 & $p<0.00001^{*}$ & $p \leq 0.2602$ \\
\hline \multicolumn{7}{|l|}{ Female } \\
\hline Control & 50 & 19.0 & & & & \\
\hline \multicolumn{7}{|l|}{ Female } \\
\hline Control & 50 & 18.5 & & & & \\
\hline $\mathrm{RV}$ & 50 & 24.5 & 32.4 & 8.7 & $p=0.0032^{*}$ & $p \leq 0.4553$ \\
\hline \multirow[t]{2}{*}{ PJ } & 50 & 27.0 & 45.9 & 30.3 & $p<0.00001^{*}$ & $p \leq 0.2856$ \\
\hline & $n$ & $\begin{array}{l}\text { Median survival time } \\
\text { (MST) in hours }\end{array}$ & $\begin{array}{l}\% \text { MST } \\
\text { increase }\end{array}$ & $\begin{array}{l}\% \text { of surviving } \\
\text { flies }\end{array}$ & ANOVA & $t$-test \\
\hline \multicolumn{7}{|c|}{ Resistance against infection } \\
\hline \multicolumn{7}{|l|}{ Male } \\
\hline Control & 50 & 4.88 & & 41 & $p=0.0013^{*}$ & \\
\hline RV & 50 & $>6$ & $>23$ & 55 & & $p \leq 0.05^{*}$ \\
\hline PJ & 50 & $>6$ & $>23$ & 64 & & $p \leq 0.05^{*}$ \\
\hline \multicolumn{7}{|l|}{ Female } \\
\hline Control & 50 & 3.82 & & 38 & $p=0.0012^{*}$ & \\
\hline RV & 50 & $>5$ & $>31$ & 59 & & $p \leq 0.05^{*}$ \\
\hline PJ & 50 & $>5$ & $>31$ & 78 & & $p \leq 0.05^{*}$ \\
\hline
\end{tabular}

*Significant. 


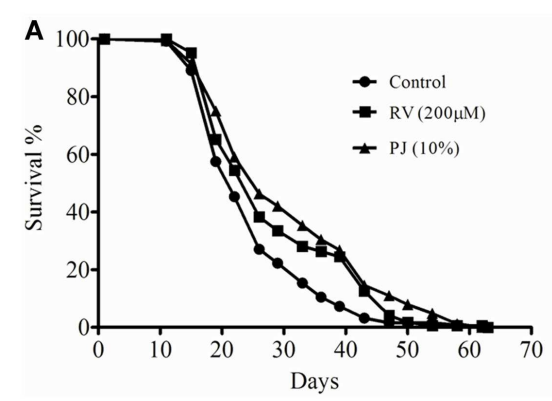

FIGURE 2 | (A). Effect of pomegranate juice supplementation on Drosophila longevity. Male and female mixed culture flies were fed with $10 \%$ PJ $(n=164)$ or $200 \mu \mathrm{M} \mathrm{RV}(n=167)$. A basal medium control $(n=247)$ was also maintained. Log rank test indicated significant $(p<0.00001)$ increase in the fly survival by both PJ and RV (Table 1). But PJ increased the median survival by $19.23 \%$ while it was $11.05 \%$ in the RV group. (B) Effect of $10 \%$

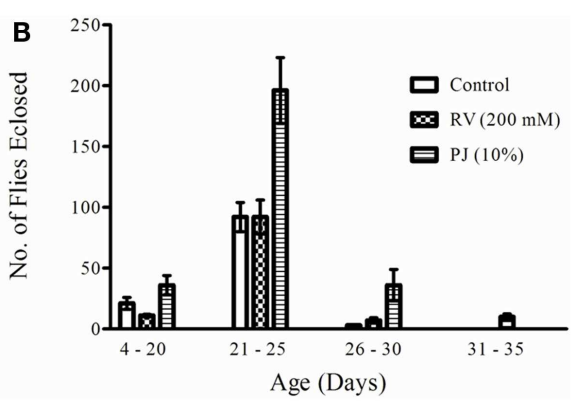

pomegranate juice supplementation on Drosophila fecundity at different age. The bars represent the total number of flies enclosed from each group at different age with SD. PJ group showed significant increase in the number of off-springs produced $\left({ }^{*} p<0.0001\right)$. The graph also indicates an extended reproductively viable phase in the PJ fed flies. No significant difference in fecundity was observed with RV and control groups.

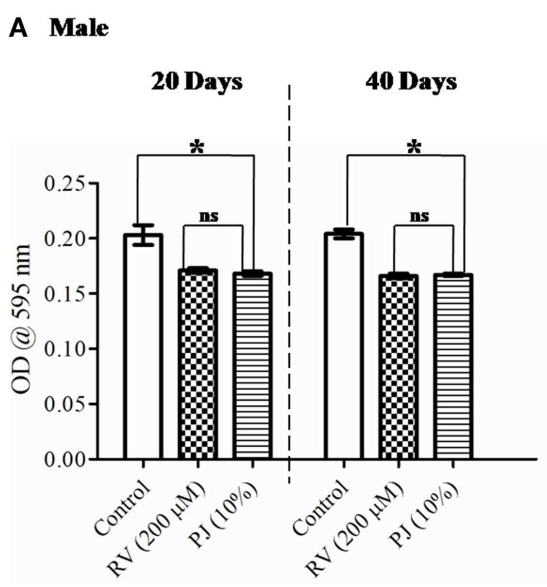

FIGURE 3 | Gustatory assay with PJ (10\%) or RV (200 $\mu \mathrm{M})$ supplementation in $\mathbf{2 0}$ and $\mathbf{4 0}$ days old Drosophila flies. (A) Male flies in the PJ and RV group were found to have reduced feed intake when compared

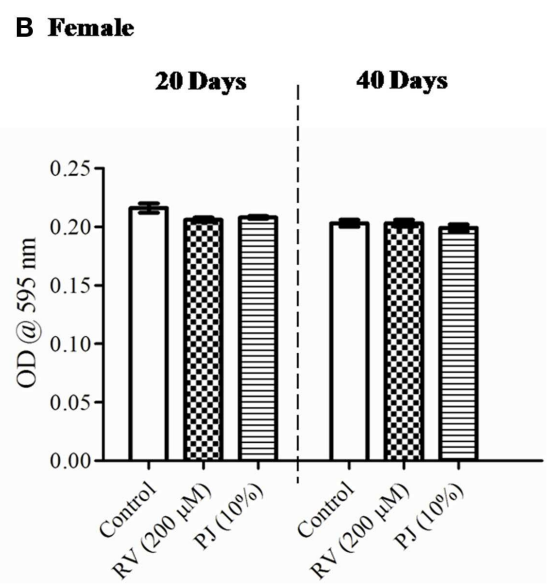

to control (* $p<0.05)$. However, there was no difference in food intake by PJ and RV groups. (B) Female flies did not show any significant difference in the food intake on both the days tested (ns, not significant).
D. melanogaster (30). Bass et al. (31) has reported that RV enhances lifespan by dietary restriction in Drosophila and C. elegans. To ensure that the observed changes in the lifespan were due to PJ or RV supplementation and not because of starvation or dietary restriction, gustatory assay was performed, quantifying the food intake on 20- and 40-day-old flies using bromophenol blue dye. While there was no significant difference observed between PJ and $\mathrm{RV}$ fed groups, the feed intake was lower in both these groups as compared to that in the control $(p<0.05)$, particularly in the male flies (Figure 3A). This difference was not significant in the female flies. The feed intake of 40-day-old female flies was similar in control, PJ, and RV groups (Figure 3B). Also, there was a significant difference $(p<0.05)$ in food intake between the male and female flies in both PJ and RV groups (Figure 3).

\section{PJ ENHANCES TOLERANCE TO FREE RADICAL INDUCED STRESS}

Ayurveda claims that Rasayanas improve the stress resistance capacity of organisms. Both $\mathrm{H}_{2} \mathrm{O}_{2}$ and paraquat produce free radicals and are lethal to flies. A significant $(p<0.00001)$ increase in the post $\mathrm{H}_{2} \mathrm{O}_{2}$ and paraquat exposure survival time was observed in both PJ and RV supplemented flies when compared to the control (Figures 4 and 5; Table 1). Analysis of median survival indicated an average $50 \%$ increase over the control group in both male and female flies in PJ. Though, RV was also found to protect against the free radical induced stress, its level of protection showed only about 30-35\% increase in survival (Figures 4 and 5).

\section{PJ PROTECTS DROSOPHILA FROM CANDIDA ALBICANS INFECTION}

Drosophila melanogaster has been used as a model to study hostfungal interaction and pathogenicity (32). Glittenberg et al. (33) established Drosophila as an alternative model for investigating the pathogenicity of $C$. albicans. Literature indicates that Rasayanas have immunomodulatory (Vyadhikshamatwa) potential (10). As proof of principle, we evaluated if PJ substitution offered protection to the flies from death because of $C$. albicans infection. The survival curve indicated a significant $(p \leq 0.05)$ protective effect of 

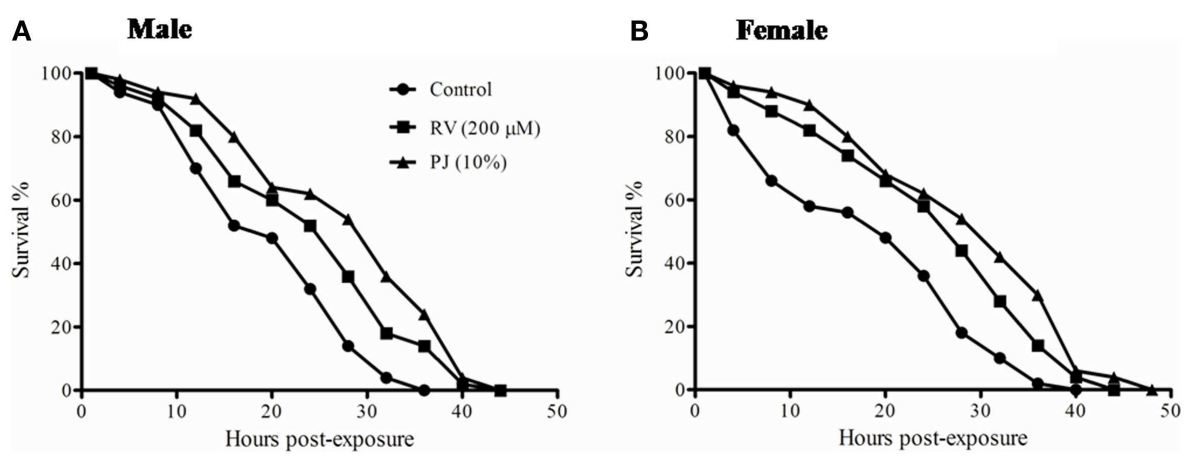

FIGURE 4 | Survival against hydroxyl radical $\left(\mathrm{OH}^{*}\right)$ produced by hydrogen peroxide exposure in 20 days old Drosophila. Both male (A) and female (B) flies fed with PJ (10\%) showed approximately $50 \%$ increase in median survival ( $p<0.00001)$ (Table 1). RV (200 $\mu$ M) fed flies showed only about $30-35 \%$ increase in median survival.

A

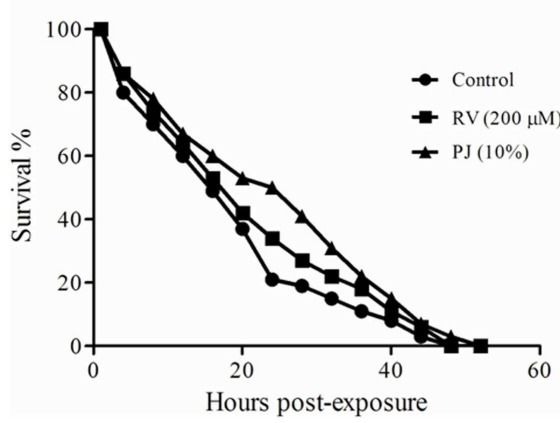

FIGURE 5 | Survival against superoxide radical ( $0 *$ ) produced by paraquat exposure in $\mathbf{2 0}$ days old Drosophila. (A) PJ (10\%) group showed $54 \%$ increase in median survival $(p<0.00001)$ while RV $(200 \mu \mathrm{M})$ could
B Female

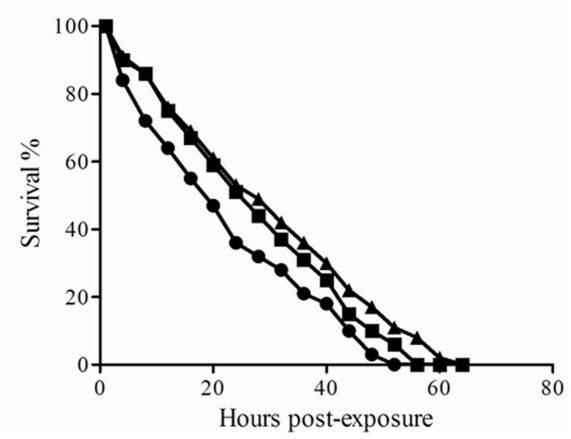

increase only by $9.6 \%$ (Table 1). (B) Female flies fed with PJ showed $45.9 \%$ increase in median survival $(p<0.00001)$ while RV was able to increase the median survival by $32.4 \%$.
PJ until $144 \mathrm{~h}$ post-infection. Even though, RV too protected the flies (>50\%) from C. albicans infection, the magnitude of protection by PJ (>70\%) was significantly higher. The control flies had only 40\% survival (Figure 6). While the magnitude of protection differs with male and female flies, the protection trend was similar.

\section{PJ PROMOTES SUSTAINED PHYSICAL PERFORMANCE IN D. MELANOGASTER}

The success of healthy living lies in retention of normal physical activity. Rasayanas like pomegranate are said to improve energy, strength, and stamina (Balya) of organisms. The physical performance of the flies was measured using the climbing or negative geo-taxis assay. This assay was performed with 20 and 40 days old male and female flies fed on media supplemented with PJ or RV. Even though, significant difference among the groups was not observed in the percentage of flies climbing on 20th day, the PJ group retained the physical activity even on day 40 (aged flies), while the other groups showed severe decline $(p<0.05)$ (Figure 7). Thus, PJ was found to sustain good physical activity in terms of negative geo-taxis climbing activity during aging.

\section{DISCUSSION}

Developing scientifically validated, culturally acceptable, appropriate guidelines for healthy eating is recognized as one of the thrust areas for reducing age-related functional decline (1). An Australian longitudinal analysis to guide health promotion concludes that health, life style, and gender influences healthy aging (34). In Ayurveda, Rasayanas are said to improve immunity, impart vitality, are good aphrodisiacs and are considered to delay the aging process $(8,10)$. Pomegranate has been mentioned in Ayurveda as a Rasayana with the above properties, which was the reason for selecting the fruit for our study. We have shown that PJ fed flies live and reproduce longer (Figure $2 \mathbf{B}$ ), are better protected against stress and infection (Figures 4-6) and are active even when aged (Figure 7). According to the antagonistic pleiotrophy theory, higher levels of reproduction are negatively correlated with survival. This concept of "trade-off" between longevity and reproduction or "cost of reproduction" has been widely accepted and demonstrated in a number of experimental studies (17). However, our observation in the current experimental setup indicated that $\mathrm{PJ}$ is capable of simultaneously enhancing fertility as well as 

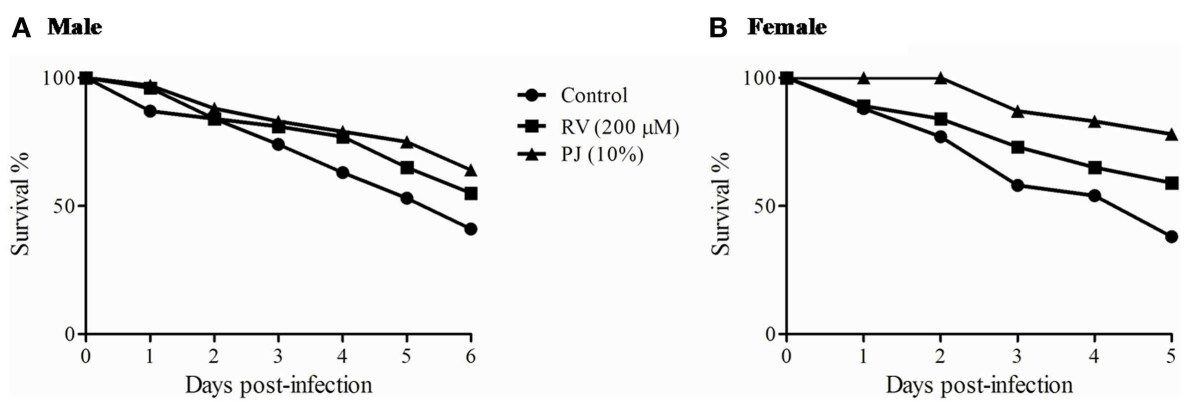

FIGURE 6 | Pomegranate protects Drosophila from Candida albicans infection. Twenty days old male (A) and female (B) flies fed with PJ, RV, or control diet were infected with Candida albicans. In both the sexes, flies fed with PJ showed a significantly $(p \leq 0.05)$ better survival response than the control and RV fed.

\section{A Male}

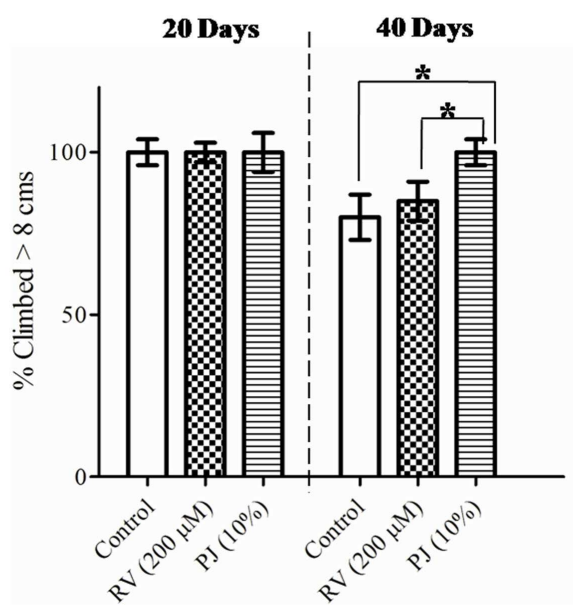

FIGURE 7 | Sustained climbing performance of Drosophila flies fed with PJ. (A) Twenty days old male flies did not show any difference in their climbing performance irrespective of feed, but 40 days old PJ fed flies were able to retain their physical performance $(p<0.0001)$ then the RV and control

\section{B Female}

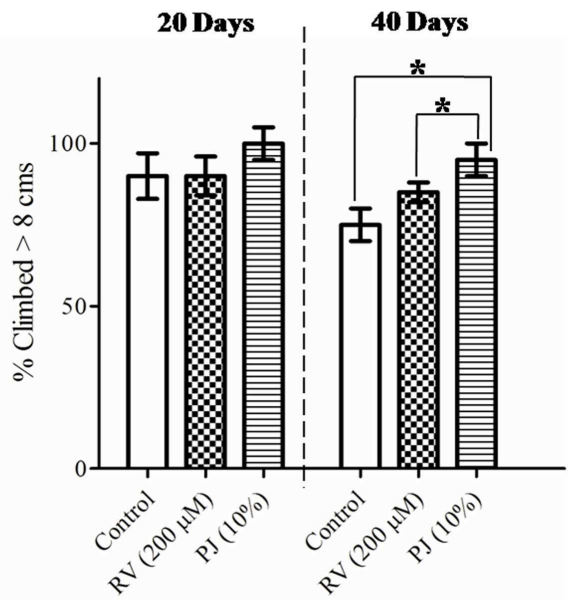

group. (B) Female flies at both 20 and 40 days showed significantly higher $(p<0.0001)$ climbing performance then the RV and control group. PJ group was able to resist the age-related physical activity decline observed in the RV and control groups. survival. Reduction in the fertility of individuals has been considered as one of the reasons for accelerated graying in population (1). Ayurvedic texts indicate Rasayanas as holistic interventions that reduce age-related senility while maintaining physical and biological performance of the organism (7). This study opens up the scope of scientifically exploring Rasayanas to identify methods and to counter the burning contemporary issue of healthy aging.

Studies have shown that elevated resistance to various environmental stresses, including oxidative stress is one of the phenotypes of long lived drosophila mutants (22). When the protecting ability of PJ and RV was assessed against $\mathrm{H}_{2} \mathrm{O}_{2}$ and paraquat induced oxidative stress, we observed that $\mathrm{PJ}$ could play a role in ameliorating the free radical induced damage, which is one of the reasons for aging. The anti-oxidant potential of pomegranate demonstrated by Faria and Calhau (12) support our findings. Cranberry extract, apple polyphenols, curcumin, are some of the fruits and herbs that have been reported to impart protection against $\mathrm{H}_{2} \mathrm{O}_{2}$ and paraquat induced free radicals in Drosophila $(19,22,28)$. Stress not only affects older individuals but it is also predisposed to cause premature aging (35). Free radicals and oxidative stress are increasingly being accepted as a major player in several disease processes and accelerated aging (36). Flavonoids and polyphenols in fruits like cranberry and blueberry have been shown to protect cells from oxidative stress induced damage caused by proinflammatory mediators (37). The polyphenol content in pomegranate may be responsible for imparting resistance against free radical induced stress (26). While fruit powders of blueberry and cranberry impart health benefits to flies at $2-3 \% \mathrm{w} / \mathrm{v}$, orange juice was found to be effective at higher concentrations $(3-50 \% \mathrm{v} / \mathrm{v})$ $(19,20)$. Purified apple polyphenols $(10 \mathrm{mg} / \mathrm{ml}$ of fly diet $)$ were found to significantly enhance the health and life-span of flies. The concentration of polyphenols was estimated to be $0.4 / 100 \mathrm{~kg}$ of apple pomace (22). 
Drosophila, although devoid of an adaptive immune system, harbors an innate immune response with striking similarities to the mammalian defense mechanisms. The Drosophila innate immune response uses pattern recognition receptors to activate phagocytosis by plasmatocytes, proteolytic clotting cascades in the hemolymph and production of specific antimicrobial peptides (AMPs) in the fly fat body (32). The expression of specific antifungal peptides is controlled by a signaling pathway orchestrated by the toll-like receptors (TLR) similar to human beings (38). The exact mechanism by which PJ exerted protection to the flies is yet to be elucidated. Infectious diseases have been posing a major threat to the elderly population because of age-related decline in immune functions (39). Such conditions can be overcome by use of immune-stimulators. Several Rasayanas like pomegranate can be simple interventions to enhance immunity. Drosophila has been established as a model to also study age-related impairment in physical movement (40). Present study indicates that pomegranate feeding can sustain physical movement in aged flies. The extended reproductive phase (Figure 2B) and sustained physical activity (Figure 7) in the flies can be considered as direct indicators of healthy life-span.

The current study corroborates earlier reports that RV enhances lifespan in Drosophila $(18,31,41)$. In this study, we have observed that RV increased median life-span of Drosophila by $11 \%$ (Figure 2A). RV substitution imparted comparatively lesser protective ability against $\mathrm{H}_{2} \mathrm{O}_{2}$, paraquat, and infection stress to the flies when compared to PJ fed group. RV did not significantly alter the fecundity of the flies as compared to the control. Estimation of the feed intake showed a slightly reduced feed intake quantity (Figure 3) in both PJ and RV supplemented groups when compared to the control. Earlier reports also indicate the possible dietary restriction by RV (31). In our experiments, this observation was more marked in the male flies than in the females. Ayurveda literature on pomegranate mentions that pomegranate provides satiation (Trupti), i.e., the organism is satisfied with lesser intake of food when supplemented with PJ $(15,16)$. While there was no significant difference in the feed intake between RV and PJ fed groups, the latter outperformed the former in all life-span and health-span parameters tested.

Free radical induced stress tolerance, improved infection survival, and climbing performance assays show that, pomegranate supplementation helps in reducing age-related functional decline in the flies. This study justifies the Ayurvedic claims that pomegranate is a Rasayana by being a multi-functional health promoter in fruit fly model. Given the exploratory nature of our study and the large number of comparisons made, the results are certainly very interesting; and it has opened up avenue for further research. Further research needs to be done to study the effectiveness of PJ supplementation in human beings at the community setting. Scientific exploration of other Rasayanas as quality-of-life enhancers is a potential area for research. Studying the mode of action of PJ, identification of bioactive components and molecular targets would be of value to understanding aging and anti-aging process.

Drosophila has been a strong candidate as a model in contemporary aging research. D. melanogaster has also been used as a model by researchers to study Rasayanas. An insect specific Rasayana was developed that was found to increase lifespan by
50\% in Drosophila model (24). Dwivedi et al. (25) reported that Amalaki Rasayana, an herbal formulation and Rasa-Sindoor an organo-metallic derivative of mercury were capable of increasing life-span and fecundity in fruit flies.

Aging population and the associated physical, physiological, and neurological deficiencies are being recognized as the major challenges for healthcare in the twenty-first century. While people are living longer; they are aging prematurely (42). Scientific exploration of Rasayana can provide affordable ways to delay aging and maintain good health.

\section{AUTHOR CONTRIBUTIONS}

Padmavathy Venkatasubramanian conceptualized the transdisciplinary research methodology, obtained fund support, and edited the manuscript. Upendra Nongthomba designed Drosophila based experiments, analyzed results, and edited manuscript. Subramani Paranthaman Balasubramani performed the experiments, analyzed, interpreted results, prepared, and edited the manuscript. Jayaram Mohan maintained the Drosophila stocks and assisted Subramani Paranthaman Balasubramani in experimentation. Esha Patnaik and Chatterjee Arunita standardized the infection and stress experimentation protocol and analysis of results. Subrahmanya Kumar Kukkupuni provided the Ayurveda knowledge.

\section{ACKNOWLEDGMENTS}

Padmavathy Venkatasubramanian, Subramani Paranthaman Balasubramani, and Subrahmanya Kumar Kukkupuni wish to thank the Department of Science and Technology, Government of India for the financial support received under the Drugs and Pharmaceuticals Research Program (DPRP) scheme. Thanks to Dr. Venugopalan Nair for his Ayurvedic inputs and Prof. Nagarajan for his suggestions. Authors wish to thank Prof. Parag Sadhale (late) for providing C. albicans strain. Thanks to Prof. P. Kondaiah, MRDG and The Director, Indian Institute of Science for extending the facility. The facility and encouragement provided by Shri. Darshan Shankar, FRLHT are thankfully acknowledged.

\section{REFERENCES}

1. World Health Organisation. Active Ageing: A Policy Framework. Geneva: Noncommunicable Disease Prevention and Health Promotion, Ageing and Life Course (2002). WHO/NMH/NPH/02.8. Available from: http://whqlibdoc.who. int/hq/2002/who_nmh_nph_02.8.pdf

2. Arai H, Ouchi Y, Yokode M, Ito H, Uematsu H, Eto F, et al. Towards the realization of a better aged society: messages from gerontology and geriatrics. Geriatr Gerontol Int (2012) 12:16-22. doi:10.1111/j.1447-0594.2011.00776.x

3. Chatterji S, Kowal P, Mathers C, Naidoo N, Verdes E, Smith JP, et al. The health of aging populations in China and India. Health Aff (2008) 27:1052-63. doi:10.1377/hlthaff.27.4.1052

4. Avendano M, Glymour MM, Banks J, Mackenbach JP. Health disadvantage in US adults aged 50 to 74 years: a comparison of the health of rich and poor Americans with that of Europeans. Am J Public Health (2009) 99:540-8. doi:10.2105/AJPH.2008.139469

5. National Health Services. From Illness to Wellness - Achieving Efficiencies and Improving Outcomes. London: NHS Confederation (2011). Available from: http://www.nhs-chaplaincy-spiritualcare.org.uk/NationalHealthService/ illness_to_wellness_241011.pdf

6. Daubenmier JJ, Weidner G, Sumner MD, Mendell N, Merritt-Worden T, Studley J, et al. The contribution of changes in diet, exercise, and stress management to changes in coronary risk in women and men in the multisite cardiac lifestyle intervention program. Ann Behav Med (2007) 33:57-68. doi:10.1207/s15324796abm3301_7 
7. Udapa MH. Comprehensive Kaayachikitsa and Principles of Ayurveda. Bangalore: Laveena Publications (2004).

8. Sharma PV, editor. Susruta-Samhita. (Vol. 1). Varanasi: Chaukhambha Visvabharati (2004).

9. Singh RH, Narsimhamurthy K, Singh G. Neuronutrient impact of ayurvedic rasayana therapy in brain aging. Biogerontology (2008) 9:369-74. doi:10.1007/ s10522-008-9185-z

10. Balasubramani SP, Venkatasubramanian P, Kukkupuni SK, Patwardhan B. Plantbased rasayana drugs from ayurveda. Chin J Integr Med (2011) 17:88-94. doi:10.1007/s11655-011-0659-5

11. Jurenka JS. Therapeutic applications of pomegranate (Punica granatum L.): a review. Altern Med Rev (2008) 13:128-44.

12. Faria A, Calhau C. The bioactivity of pomegranate: impact on health and disease. Crit Rev Food Sci Nutr (2011) 51:626-34. doi:10.1080/ 10408391003748100

13. Johanningsmeier SD, Harris GK. Pomegranate as a functional food and nutraceutical source. Annu Rev Food Sci Technol (2011) 2:181-201. doi:10.1146/ annurev-food-030810-153709

14. Mahdihassan S. Outline of the beginnings of alchemy and its antecedents. Am J Chin Med (1984) 12:32-42. doi:10.1142/S0192415X84000039

15. Chunekar KC, editor. Bhavaprakasha Nighantu. Varanasi: Chaukhambha Bharati Academy (2004).

16. Dash B. Materia Medica of Ayurveda based on Madanapala's Nighantu. New Delhi: B. Jain Publishers (P) Ltd (1991).

17. Iliadi KG, Knight D, Boulianne GL. Healthy aging - insights from Drosophila. Front Physiol (2012) 3:106. doi:10.3389/fphys.2012.00106

18. Johnson SC, Rabinovitch PS, Kaeberlein M. mTOR is a key modulator of aging and age-related disease. Nature (2013) 493:338-45. doi:10.1038/ nature11861

19. Wang C, Yolitz J, Alberico T, Laslo M, Sun Y, Wheeler CT, et al. Cranberry interacts with dietary macronutrients to promote healthy aging in Drosophila. J Gerontol A Biol Sci Med Sci (2014) 69:945-54. doi:10.1093/gerona/glt161

20. Peng C, Zuo Y, Kwan KM, Liang Y, Ma KY, Chan HY, et al. Blueberry extract prolongs lifespan of Drosophila melanogaster. Exp Gerontol (2012) 47:170-8. doi:10.1016/j.exger.2011.12.001

21. Fernandez-Bedmar Z, Anter J, de La Cruz-Ares S, Munoz-Serrano A, AlonsoMoraga A, Perez-Guisado J. Role of citrus juices and distinctive components in the modulation of degenerative processes: genotoxocity, antigenotoxicity, cytotoxicity and longevity in Drosophila. J Toxicol Environ Health A (2011) 74:1052-66. doi:10.1080/15287394.2011.582306

22. Peng C, Chan HY, Huang Y, Yu H, Chen ZY. Apple polyphenols extend the mean lifespan of Drosophila melanogaster. J Agric Food Chem (2011) 59:2097-106. doi:10.1021/jf1046267

23. Bahadorani S, Hilliker AJ. Cocoa confers life span extension in Drosophila melanogaster. Nutr Res (2008) 28:377-82. doi:10.1016/j.nutres.2008.03.018

24. Priyadarshini S, Ashadevi JS, Nagarjun V, Prasanna KS. Increase in Drosophila melanogaster longevity due to rasayana diet: preliminary results. J Ayurveda Integr Med (2010) 1:114-9. doi:10.4103/0975-9476.65085

25. Dwivedi V, Anandan EM, Mony RS, Muraleedharan TS, Valiathan MS, Mutsuddi M, et al. In vivo effects of traditional ayurvedic formulations in Drosophila melanogaster model relate with therapeutic applications. PLoS One (2012) 7:e37113. doi:10.1371/journal.pone.0037113

26. Wang R, Ding Y, Liu R, Xiang L, Du L. Pomegranate: constituents, bioactivities and pharmacokinetics. Fruits Veg Cereal Sci Biotech (2010) 4:77-87.

27. Medjakovic S, Jungbauer A. Pomegranate: a fruit that ameliorates metabolic syndrome. Food Funct (2013) 4:19-39. doi:10.1039/c2fo30034f

28. Lee KS, Lee BS, Semnani S, Avanesian A, Um CY, Jeon HJ, et al. Curcumin extends life span, improves health span, and modulates the expression of age-associated aging genes in Drosophila melanogaster. Rejuvenation Res (2010) 13:561-70. doi:10.1089/rej.2010.1031

29. Apidianakis Y, Rahme LG. Drosophila melanogaster as a model host for studying Pseudomonas aeruginosa infection. Nat Protoc (2009) 4:1285-94. doi:10.1038/ nprot.2009.124
30. Grandison RC, Piper MD, Partridge L. Amino acid imbalances explains extension of lifespan by dietary restriction in Drosophila. Nature (2010) 462:1061-4. doi:10.1038/nature08619

31. Bass TM, Weinkove D, Houthoofd K, Gems D, Partridge L. Effects of resveratrol on lifespan in Drosophila melanogaster and Caenorhabditis elegans. Mech Ageing Dev (2007) 128:546-52. doi:10.1016/j.mad.2007.07.007

32. Alarco AM, Marcil A, Chen J, Suter B, Thomas D, Whiteway M. Immunedeficient Drosophila melanogaster: a model for the innate immune response to human fungal pathogens. J Immunol (2004) 172:5622-8. doi:10.4049/ jimmunol.172.9.5622

33. Glittenberg MT, Silas S, MacCallum DM, Gow NA, Ligoxygakis P. Wildtype Drosophila melanogaster as an alternative model system for investigating the pathogenicity of Candida albicans. Dis Model Mech (2011) 4:504-14. doi:10.1242/dmm.006619

34. Kendig H, Browning CJ, Thomas SA, Wells Y. Health, lifestyle and gender influences on aging well: an Australian longitudinal analysis to guide health promotion. Front Public Health (2014) 2:70. doi:10.3389/fpubh.2014.00070

35. Quinlan J, Tu MT, Langlois EV, Kapoor M, Ziegler D, Fahmi H, et al. Protocol for a systematic review of the association between chronic stress during the life course and telomere length. Syst Rev (2014) 3:40. doi:10.1186/20464053-3-40

36. Lagouge M, Larsson NG. The role of mitochondrial DNA mutations and free radicals in disease and ageing. J Intern Med (2013) 273:529-43. doi:10.1111/ joim. 12055

37. Khurana S, Venkataraman K, Hollingsworth A, Piche M, Tai TC. Polyphenols: benefits to the cardiovascular system in health and in aging. Nutrients (2013) 5:3779-827. doi:10.3390/nu5103779

38. Chamilos G, Lionakis MS, Lewis RE, Lopez-Ribot JL, Saville SP, Albert ND, et al. Drosophila melanogaster as a facile model for large-scale studies of virulence mechanisms and antifungal drug efficacy in Candida species. J Infect Dis (2006) 193:1014-22. doi:10.1086/500950

39. Montecino-Rodriguez E, Berent-Maoz B, Dorshkind K. Causes, consequences and reversal of immune system in aging. J Clin Invest (2013) 123:958-65. doi:10.1172/JCI64096

40. Jones MA, Grotewiel M. Drosophila as a model for age-related impairment in locomotor and other behaviors. Exp Gerontol (2011) 46:320-5. doi:10.1016/j. exger.2010.08.012

41. Kaeberlein M. Resveratrol and rapamycin: are they anti-aging drugs? Bioessays (2010) 32:96-9. doi:10.1002/bies.200900171

42. World Health Organization and US National Institute of Aging. Global Health and Aging. Washington, DC (2011). NIH Publication No. 117737. Available from: http://www.who.int/ageing/publications/global_health. pdf?ua $=1$

Conflict of Interest Statement: The authors declare that the research was conducted in the absence of any commercial or financial relationships that could be construed as a potential conflict of interest.

Received: 01 August 2014; accepted: 05 November 2014; published online: 16 December 2014

Citation: Balasubramani SP, Mohan J, Chatterjee A, Patnaik E, Kukkupuni SK, Nongthomba $U$ and Venkatasubramanian P (2014) Pomegranate juice enhances healthy lifespan in Drosophila melanogaster: an exploratory study. Front. Public Health 2:245. doi: $10.3389 /$ fpubh.2014.00245

This article was submitted to Environmental Health, a section of the journal Frontiers in Public Health.

Copyright (C) 2014 Balasubramani, Mohan, Chatterjee, Patnaik, Kukkupuni, Nongthomba and Venkatasubramanian. This is an open-access article distributed under the terms of the Creative Commons Attribution License (CC BY). The use, distribution or reproduction in other forums is permitted, provided the original author(s) or licensor are credited and that the original publication in this journal is cited, in accordance with accepted academic practice. No use, distribution or reproduction is permitted which does not comply with these terms. 